\section{International Scientific Journal Theoretical \& Applied Science}

\author{
p-ISSN: 2308-4944 (print) e-ISSN: 2409-0085 (online) \\ Year: 2015 Issue: 12 Volume: 32 \\ Published: $30.12 .2015 \quad$ http://T-Science.org
}

SECTION 4. Computer science, computer engineering and automation.
Vjacheslav Vadimovich Syskov Ph. D. Tech. Sc., Chief Researcher, Department of

Modeling and Control Systems Joint Stock Company «Central research institute of economy, informatics and control systems», Russia

slavacestmoi@gmail.com

Vadim Vladimirovich Borisov Dr. Tech. Sc., Professor, Branch of the National Research University «MPEI» in Smolensk, Russia vadim.v.borisov@mail.ru

\title{
THE METHOD OF OPERATIONAL MANAGEMENT OF WORK AT REALIZATION OF COLLECTIVE ACTIVITY IN ORGANIZATIONAL AND TECHNICAL SYSTEMS
}

\begin{abstract}
The method of operational management of work in organizational and technical systems that enables intelligent control of manpower, materials and the equipment in the course of business processes is offered. The method consists in the analysis of changes in performance and efficiency indicators, determined at execution of formalized of activity models, and monitoring associated with these indicators of operational risks, taking into account various negative events arising from the realization of collective activity on the basis of these activity models. The reported study was partially supported by RFBR, research project No. 14-07-00338_a, and by Ministry of Education of Russia, project No. 2493 in basic part of the state task No. 2014/123.

Key words: business process, collective activity, business process modeling, business process management, process indicators, resources allocation, management of work, operational risks, risk management.

Language: Russian

Citation: Syskov VV, Borisov VV (2015) THE METHOD OF OPERATIONAL MANAGEMENT OF WORK AT REALIZATION OF COLLECTIVE ACTIVITY IN ORGANIZATIONAL AND TECHNICAL SYSTEMS. ISJ Theoretical \& Applied Science 12 (32): 10-27.

Soi: http://s-o-i.org/1.1/TAS-12-32-3 Doi: crossef http://dx.doi.org/10.15863/TAS.2015.12.32.3

\section{МЕТОД ОПЕРАТИВНОГО УПРАВЛЕНИЯ РАБОТОЙ ПРИ РЕАЛИЗАЦИИ КОЛЛЕКТИВНОЙ ДЕЯТЕЛЬНОСТИ В ОРГАНИЗАЦИОННО-ТЕХНИЧЕСКИХ СИСТЕМАХ}

Аннотация: Предложен метод оперативного управления работой в организационно-технических системах, который позволяет осущзествлять интеллектуальное регулирование трудовых ресурсов, материалов и оборудования в прочессе выполнения бизнес-процессов. Суть метода заключается в анализе изменения показателей производительности и эффективности, определяемых при исполнении формализованных моделей деятельности, и контроле связанных с этими показателями операционных рисков, учитывающих отдельные негативные события, возникающие при реализации коллективной деятельности на основе этих моделей деятельности.

Ключевые слова: бизнес-процесс, коллективная деятельность, моделирование бизнес-проиессов, процессное управление, показатели процессов, распределение ресурсов, управление деятельностью, операционные риски, управление рисками.
\end{abstract}

\section{Введение}

Задача реализации коллективной деятельности в организационно-технических системах главным образом связана с решением проблем организации коллективной деятельности, оперативного управления работой, а также выработки обоснованных управленческих решений при ее выполнении.
Данная статья посвящена решению задачи оперативного управления работой при реализации коллективной деятельности, в том числе выработки решений по распределению трудовых и других видов ресурсов, необходимых на наиболее трудных ее участках, где возникают риски нарушения регламента выполнения.

Актуальность данной темы обусловлена тем, что в настоящее время в системах процессного управления (ВРМ-системах, Business Process 
Management Systems) практически не уделяется внимание решению задач, связанным с оперативным управлением работой. В некоторых системах к ним можно отнести возможности по отображению числа выполнений процессов с тем или иным исходом, продолжительности процессов и распределения заданий между участниками деятельности, а также информирования о работах с наибольшими задержками (выявление узких мест в модели процесса) [1, с. 31].

При этом в большинстве ВРМ-систем практически не обеспечивается автоматизация оперативного управления работой, в частности не поддерживается анализ возможности возникновения негативных ситуаций или нарушения регламента выполнения процессов, расходования различных видов ресурсов при этом. Из этого возможно сделать вывод о недостаточности существующих возможностей для оперативного управления работой в современных системах процессного управления. Поэтому особенности, связанные с автоматизацией мониторинга хода деятельности, оперативного управления работой и оценкой операционных рисков (ОР) требуют развития, в первую очередь, в части совершенствования методического аппарата.

Общие вопросы управления рисками при процессном управлении и их классификация рассмотрены в [2], где выделены риски, связанные с отсутствием или злоупотреблением требуемых ресурсов или навыков участников деятельности, ошибками или неверной работой оборудования или сотрудников, а также ошибками управления или неспособностью осуществлять изменения хода процесса.

\section{Назначение метода оперативного управления работой}

Целью данной статьи является разработка метода, обеспечивающего интеллектуальное оперативное управление работой при реализации коллективной деятельности в организационнотехнических системах посредством целеполагания, анализа ОР и выдачи рекомендаций по распределению ресурсов в таких ситуациях на уровне их владельцев.

Метод оперативного управления работой должен устанавливать порядок действий по мониторингу деятельности на основе системы показателей и контролю ОР посредством отслеживания нарушений ее выполнения с целью выработки решений по регулированию трудовых ресурсов, расхода материалов и оборудования, а также анализу результатов такого управления.

При этом именно бизнес-процесс является основным объектом изучения при управлении OP, а его формализованное описание - основным условием эффективного управления ОР, начиная с определения самих типов рисков в каждом из процессов [3].

Интеллектуальность

оперативного управления работой при этом подразумевает учет целей, контроль ОР в случае отклонения показателей процессов от целевых значений, оценку OP за счет расчета возможности возникновения негативных событий, а также выбор для всех видов ресурсов соответствующих корректирующих мероприятий на наиболее трудных участках деятельности. Эти мероприятий могут заключаться в определении порядка использования, управлении расходования или замене типа ресурса.

\section{Описание метода оперативного управления работой при реализации коллективной деятельности}

В общем виде оперативное управление работой в теории процессного управления реализуется на основе системы показателей и включает этапы, где [4, с. 44]:

- планируется деятельность компании и процессов, в том числе устанавливаются целевые значения по процессам на краткосрочную перспективу;

- выполняется организация деятельности, владельцам деятельности выделяются необходимые ресурсы;

- осуществляется мониторинг хода и результаты процессов;

- анализируется эффективность и результативность деятельности организации и процессов.

С учетом введения шагов для управления $\mathrm{OP}$, для оперативного управления работой при реализации коллективной деятельности, предлагается метод, который включает этапы как показано на рисунке 1. 


\begin{tabular}{|c|c|c|c|c|c|c|}
\hline Impact Factor: & $\begin{array}{l}\text { ISRA (India) } \\
\text { ISI (Dubai, UAE } \\
\text { GIF (Australia) } \\
\text { JIF }\end{array}$ & $\begin{array}{l}=1.344 \\
=0.829 \\
=0.564 \\
=1.500\end{array}$ & $\begin{array}{l}\text { SIS (USA) } \\
\text { PИНЦ (Russia) } \\
\text { ESJI (KZ) } \\
\text { SJIF (Morocco) }\end{array}$ & $\begin{array}{l}=0.912 \\
=0.179 \\
=1.042 \\
=2.031\end{array}$ & $\begin{array}{l}\text { ICV (Poland) } \\
\text { PIF (India) }\end{array}$ & $\begin{array}{l}=6.630 \\
=1.940\end{array}$ \\
\hline
\end{tabular}

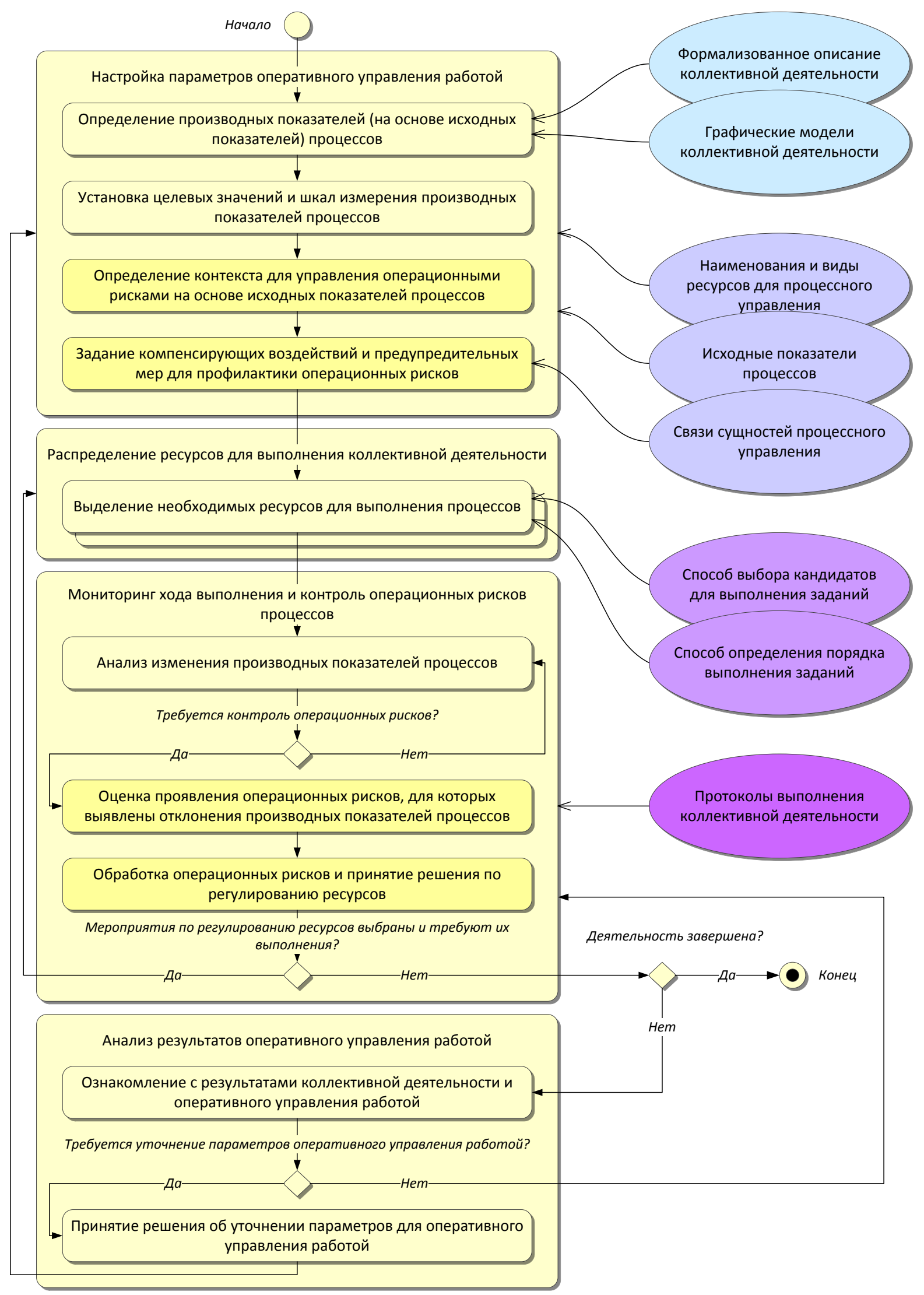

Рисунок 1 - Схема метода оперативного управления работой.

ISPC Applied technologies, 
Этап 1 выполняется заблаговременно, либо при необходимости перенастройки параметров в процессе работы (иногда). Этапы 2, 3 и 4 - в процессе оперативного управления работой и обеспечивают осуществление действий по распределению ресурсов, мониторингу хода выполнения и контролю ОР процессов, а также анализу результатов оперативного управления работой. При этом на всех этапах подразумевается участие владельца процесса.

Помимо порядка осуществления, эта схема устанавливает применение формализованного описания и графических моделей деятельности, протоколов еe выполнения, a также других элементов для определения ОР.

\section{Этап 1. Настройка параметров} оперативного управления работой

При настройке параметров оперативного управления работой предполагается выполнять действия по определению производных показателей процессов, установке целевых значений и шкал измерения этих показателей, определению контекста для управления ОР, a также заданию компенсирующих воздействий и предупредительных мер для их профилактики.

\section{Шаг 1. Определение производных} показателей процессов

Как было установлено в [5] показатели для реализации процессного управления можно разделить по происхождению их данных на исходные, которые могут быть получены при исполнении процессов, и производные, рассчитываемые на основании этих исходных показателей. Исходные и производные показатели предлагается рассматривать по категориям, описание которых приведено в таблице 1.

Таблица 1

Категории показателей процессов.

\begin{tabular}{|c|c|c|c|}
\hline & \multicolumn{2}{|c|}{ Наименование и обозначение } & Описание категории \\
\hline \multirow{2}{*}{ 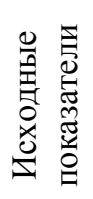 } & $I_{\text {func }}$ & $\begin{array}{l}\text { Показатели } \\
\text { функционирования }\end{array}$ & $\begin{array}{l}\text { Данные о состоянии процессов - ходе выполнения и } \\
\text { событиях потока управления }\end{array}$ \\
\hline & $I_{\text {costs }}$ & $\begin{array}{l}\text { Показатели затрат } \\
\text { ресурсов }\end{array}$ & $\begin{array}{l}\text { Характеризуют меру использования материальных ресурсов } \\
\text { (материалов, оборудования), а также трудовых ресурсов }\end{array}$ \\
\hline \multirow{3}{*}{ 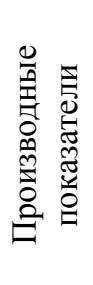 } & $I_{g e n}$ & $\begin{array}{l}\text { Обобщающие } \\
\text { показатели }\end{array}$ & $\begin{array}{l}\text { Обобщают данные о показателях функционирования и } \\
\text { затрат ресурсов (по данным одной из категорий) }\end{array}$ \\
\hline & $I_{\text {prod }}$ & $\begin{array}{l}\text { Показатели } \\
\text { производительности }\end{array}$ & $\begin{array}{l}\text { Характеризуют соотношение показателей результативности } \\
\text { и функционирования процессов }\end{array}$ \\
\hline & $I_{\text {perf }}$ & $\begin{array}{l}\text { Показатели } \\
\text { эффективности }\end{array}$ & $\begin{array}{l}\text { Характеризуют соотношение показателей затрат ресурсов и } \\
\text { результативности }\end{array}$ \\
\hline
\end{tabular}

Рассмотрим пример одного из множества взаимосвязанных процессов по приему заказа, выполняемых в некоторой организационнотехнической системе [5]. Этот процесс согласно [6] можно представить в следующем виде:

$$
\begin{aligned}
P_{\text {ordRec }}=\delta \cdot s_{\text {start }} & -P_{\text {ordHandl }} \\
& -\left(\delta \cdot s_{\text {end }, \text { notArr }}: c_{\text {notArr }} \div \delta\right. \\
& \cdot s_{\text {end,notAcc }}: c_{\text {notAcc }} \\
& \left.\div\left(P_{\text {reqSend }}-\delta \cdot s_{\text {end }}\right): c_{\text {default }}\right),
\end{aligned}
$$

где - и $\div-$ операции последовательной и альтернативной композиции заданий; PordHandl и $P_{\text {reqsend }}$ - задания по согласованию заказа и размещению заявки на его выполнение; $c_{\text {notAcc }}-$ некоторое условие, при котором заказ не может быть согласован (индивидуальные условия заказа не согласованы); $c_{n o t A r r}-$ условие, при котором коммерческое предложение по заказу не устраивает клиента; $c_{\text {default }}$ - условие, устанавливающее задание по умолчанию среди альтернативных заданий, если условия их выбора (в данном случае, $c_{\text {notAcc }}$ и $c_{\text {notArr }}$ ) не выполнены.

Для данного процесса приведем исходные показатели, которые будут использоваться для определения производных показателей.

Показатели функционирования процессов, отражающие число выполнений (экземпляров с любым исходом) и наступлений некоторого события при его выполнении в общем виде определяются, как предложено в [5]:

$$
\text { - } I_{n}=\sum_{\{i\}}\left(P_{n}^{i} \mid s_{\text {start }} \in P_{n}^{i}\right) \quad \text { - число всех }
$$
выполнений процесса (с сигналом начала); 
- $I_{n}($ per $), I_{n / U}, I_{n, e}$ - число всех выполнений $P_{n}$ соответственно за некоторый период, сотрудником $U$, а также при наступлении события $e$ (с ошибкой, не в срок);

- $I_{n, e}^{i}-$ количество всех наступлений $e$ при выполнении экземпляра процесса $P_{n}^{i}$;

- $t_{n}^{i}=\operatorname{runtime}\left(P_{n}^{i}\right) \quad$ - время выполнения экземпляра процесса $P_{n}^{i}$;

- $t_{n}^{i}($ per $), t_{n / U}^{i}, t_{n, e}^{i} \quad-$ время выполнения экземпляра процесса $P_{n}^{i}$ соответственно за некоторый период, либо определенным сотрудником, а также при наступлении некоторого события.

Для рассматриваемого примера показатели функционирования можно задать множеством:

$$
I_{\text {func }}=\left(\begin{array}{c}
I_{o R}, I_{o R, n o t A r r}, I_{o R, n o t A c c} \\
I_{o H n d}, I_{o H n d / c l r k}, I_{r S n d}, \\
t_{o H n d}^{i}, t_{o H n d / c l r k}^{i}, t_{r S n d}^{i}
\end{array}\right),
$$

где $I_{o R}$ - число всех выполнений (экземпляров) процесса $P_{\text {ordRec }} ; I_{\text {oR,notArr }}$ - число выполнений $P_{\text {ordRec }}$ c событием «предложение отклонено»; $I_{o R, n o t A c c}$ - число выполнений процесса $P_{\text {ordRec }}$ с событием «заказ не согласован»; $I_{o H n d}, I_{o H n d / c l r k}$ - число всех выполнений $P_{\text {ordHandl }}$ любыми сотрудниками или сотрудниками, замещающими должность секретаря-делопроизводителя; $I_{r S n d}-$ число выполнений процесса $P_{\text {reqsend }} ; t_{o H n d}^{i}$,

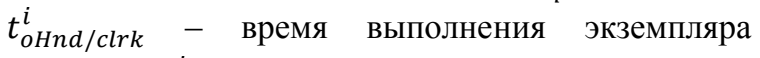
процесса $P_{\text {ordHandl }}^{i}$ любыми сотрудниками, либо секретарем-делопроизводителем; $t_{r S n d}^{i}$ - время выполнения процесса $P_{\text {reqsend }}^{i}$.

Показатели затрат ресурсов, определяющие число задействованных в процессе сотрудников, использованных материалов или единиц оборудования в общем виде задаются как:

- $N_{n}, N_{n}^{i}$ - количество сотрудников, выполняющих $P_{n}$ в целом или конкретный экземпляр $P_{n}^{i}$;

- $N_{n / E}, \quad N_{n / G}$ - количество сотрудников должности $E$ (отдела $G$ ), выполняющих $P_{n}$;

- $N m_{n}^{i}, N m_{n}$ - количество материалов любых типов, использованных в экземпляре или процессе $P_{n}$ в целом;

- $N m_{n / M}$ - количество материалов типа $M$, использованных в процессе $P_{n}$;

- $N h_{n}^{i}, \quad N h_{n} \quad$ - количество единиц оборудования, задействованных в экземпляре или процессе $P_{n}$ в целом;

- $N h_{n / H}$ - количество единиц оборудования типа $H$, задействованных в процессе $P_{n}$.

Для процесса «прием заказа» определим единственный показатель затрат ресурсов:

$$
I_{\text {costs }}=\left(N m_{o H n d / M}\right),
$$

где $N m_{o H n d} / M \quad-\quad$ количество материалов некоторого типа, расход которых учитывается среди затрат на выполнение процесса $P_{\text {ordHandl }}$.

С помощью основных математических операций над этими исходными показателями могут быть рассчитаны искомые производные показатели.

Показатели, обобщающие результаты по общему и среднему времени выполнения, доле экземпляров с каким-либо исходом, времени выполнения процессов сотрудниками, а также стоимости процессов, определяются как:

- $t_{n}=\sum_{\{i\}} t_{n}^{i}$ - общее время выполнений $P_{n}$;

- $t_{n}($ per $), t_{n / U}, \quad t_{n, e}-$ общее время выполнений процесса $P_{n}$ соответственно за некоторый период, сотрудником $U$, а также при наступлении события $e$;

- $R s t_{n}=t_{n} / I_{n}-$ среднее время выполнения процесса $P_{n}$;

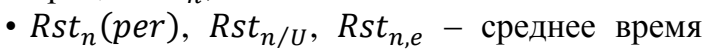
выполнения процесса $P_{n}$ соответственно за некоторый период, сотрудником $U$, а также при наступлении события $e$;

- $\operatorname{Pri}_{n}($ per $)=I_{n}($ per $) / I_{n}$ - доля выполнений экземпляров $P_{n}$ за некоторый период (относительно всех выполнений);

- $\operatorname{Pri}_{n / U}, \quad \operatorname{Pri}_{n, e} \quad$ - доля выполнений экземпляров процесса $P_{n}$ сотрудником $U$ или с некоторым событием;

- $t_{U}, t_{U}(p e r)$ - время работы сотрудника $U$ (выполнения им всех работ во всех процессах) в целом или за некоторый период;

- $V_{n / U}$ - стоимость выполнений процесса $P_{n}$ сотрудником $U$ (исходя из времени $t_{U}$ );

- $V u_{n}$ - стоимость работы всех сотрудников, участвующих в выполнении $P_{n}$ (исходя из стоимостей $V_{n / U}$ и числа выполняющих еe сотрудников);

- $V_{n / H}, \quad V_{n / M}-$ стоимость использования оборудования $H$ (исходя из времени и количества единиц оборудования) или материалов $M$ (исходя из их количества) при выполнении $P_{n}$;

- $V h_{n}, V m_{n}$ - стоимость использования в процессе $P_{n}$ всех типов оборудования (исходя из $V_{n / H}$ ) или материалов (исходя из стоимости $\left.V_{n / M}\right)$;

- $V_{n}=V m_{n}+V h_{n}+V u_{n}, \quad V_{n}($ per $)$ - общая стоимость выполнения (всех экземпляров) $P_{n}$ в целом или за некоторый период.

Для рассматриваемого примера по приему заказа обобщающие показатели можно задать множеством: 


$$
I_{g e n}=\left(\begin{array}{c}
t_{o R}, R s t_{r S n d}, R s t_{o H n d}, \\
R s t_{o H n d / c l r k}, P r i_{o R, n o t A r r}, V_{o R}
\end{array}\right)
$$

где $t_{o R}=t_{\text {oHnd }}+t_{r S n d}-$ продолжительность $P_{\text {ordRec }} ; R s t_{r S n d}$ - среднее время выполнения $P_{\text {reqSend }} ; R s t_{\text {oHnd }}, R s t_{o H n d / c l r k}$ - среднее время выполнения $P_{\text {ordHandl }}$ любыми сотрудниками или сотрудниками, замещающими должность секретаря-делопроизводителя; Pri $i_{\text {oR,notArr }}$ - доля экземпляров с отклоненным предложением относительно всех экземпляров $P_{\text {ordRec }} ; V_{o R}-$ стоимость выполнения $P_{\text {ordRec }} ; t_{\text {oHnd }}, t_{\text {oHnd/clrk }}-$ время выполнения процесса $P_{\text {ordHandl }}$ любыми сотрудниками и секретарем; $t_{r S n d}-$ время выполнения процесса $P_{\text {reqsend }}$.

Показатели производительности рассчитываются как соотношение полученного результата (представляемого показателями функционирования и обобщающими показателями) ко времени [7]:

- $\operatorname{Pru}_{n}=N_{n} / t_{n}$ - загрузка (среднее число задействованных) сотрудников в $P_{n}$;

- $\operatorname{Pru}_{n}($ per $)=N_{n}($ per $) / t_{n}($ per $) \quad$ - загрузка сотрудников при выполнении процесса $P_{n}$ за некоторый период;

- $\operatorname{Pru}_{n, e}=N_{n, e} / t_{n, e}-$ загрузка сотрудников при выполнении $P_{n}$ с некоторым событием;

- $\operatorname{Prm}_{n / M}=N m_{n / M} / t_{n}-$ расход (среднее число использованных) материалов некоторого типа при выполнении $P_{n}$;

- $P r h_{n / H}=N h_{n / H} / t_{n}-$ загрузка (среднее число задействованных единиц) оборудования некоторого типа при выполнении $P_{n}$;

- $\operatorname{Prt}_{n}=I_{n} / t_{n}-$ производительность при выполнении $P_{n}$;

- $\operatorname{Prt}_{n}($ per $), \quad \operatorname{Prt}_{n / U}, \quad \operatorname{Prt}_{n, e} \quad$ производительность при выполнении $P_{n}$ соответственно за некоторый период, либо определенным сотрудником, а также при наступлении некоторого события.

Для процесса по приему заказа показатели производительности можно задать в виде:

$$
I_{\text {prod }}=\left(\text { Prt }_{o R, \text { success }}, \operatorname{Prm}_{o H n d / M}\right),
$$

где $\quad \operatorname{Prt} t_{o R, \text { success }}=I_{o R, \text { success }} / t_{o R}$

производительность при выполнении PordRec (отражает количество согласованных заказов, по которым размещены заявки на выполнение, относительно общей продолжительности этого процесса) и $I_{o R, \text { success }}=I_{r S n d} \quad$ (по заданной модели $P_{\text {ordRec }}$ ); Prm PHnd/M $_{\text {- средний расход }}$ некоторого материала при выполнении процесса PordHandl .

Показатели эффективности рассчитываются как соотношение затрат ресурсов и полученного результата, т. е. «выступают интегральными характеристиками деятельности» [7]:

- $P f v_{n}=V_{n} / I_{n} \quad$ - средняя стоимость выполнения процесса (одного экземпляра с любым исходом) $P_{n}$;

- $P f v_{n}(p e r), \quad P f v_{n / U}, \quad P f v_{n, e}-$ средняя стоимость при выполнении процесса $P_{n}$ соответственно за некоторый период, определенным сотрудником, а также при наступлении некоторого события;

- $P f u_{n}=V u_{n} / I_{n}-$ средняя стоимость работы сотрудников при выполнении $P_{n}$;

- $P f u_{n}(p e r), \quad P f u_{n, e}$ - средняя стоимость работы сотрудников при выполнении $P_{n}$ за некоторый период с некоторым событием;

- $P f m_{n}=V m_{n} / I_{n}-$ средняя стоимость материалов (всех типов) при выполнении процесса $P_{n}$;

- $P f m_{n}(p e r), \quad P f m_{n, e}$ - средняя стоимость материалов при выполнении процесса $P_{n}$ за некоторый период с некоторым событием;

- $P f h_{n}=V h_{n} / I_{n} \quad$ - средняя стоимость использования оборудования (всех типов) при выполнении $P_{n}$;

- $P f h_{n}($ per $), \quad P f h_{n, e}$ - средняя стоимость использования оборудования при выполнении $P_{n}$ за некоторый период или с некоторым событием.

Для рассматриваемого примера определим единственный показатель эффективности:

$$
I_{\text {perf }}=\left(P f v_{o R}\right),
$$

где $P f v_{o R}=V_{o R} / I_{o R}-$ средняя стоимость выполнения $P_{\text {ordRec }}$.

Приведенные производные показатели рассчитываются исходя из реальных данных о выполнении процессов в ВРМ-системе, и необходимы для мониторинга хода выполнения деятельности и оперативного управления работой на их основе.

Далее для указания этих показателей в общих выражениях будут использоваться символы вида $Y_{n, l}$ и $Y_{n, l}^{i}$, обозначающие показатели $Y_{n, e / y}(p e r)$ и $Y_{n, e / y}^{i}(p e r)$, где $l-$ индекс некоторого показателя, учитывающего некоторое событие $e$ или учет ресурса $y$ при выполнении $P_{n, e / y}$.

\section{Шаг 2. Установка целевых значений} производных показателей процессов

Как было отмечено выше успех реализации коллективной деятельности при процессном управлении определяется исходя из оценки достижимости целей деятельности, заданных для различных ее показателей $[4$, с. 27 , с. $363 ; 8]$. В этом случае каждая цель представляет оптимальное (с точки зрения успешности 


\begin{tabular}{|c|c|c|c|c|c|c|}
\hline Impact Factor: & $\begin{array}{l}\text { ISRA (India) } \\
\text { ISI (Dubai, UAE } \\
\text { GIF (Australia) } \\
\text { JIF }\end{array}$ & $\begin{array}{l}=1.344 \\
=0.829 \\
=0.564 \\
=1.500\end{array}$ & $\begin{array}{l}\text { SIS (USA) } \\
\text { PИНЦ (Russia) } \\
\text { ESJI (KZ) } \\
\text { SJIF (Morocco) }\end{array}$ & $\begin{array}{l}=0.912 \\
=0.179 \\
=1.042 \\
=2.031\end{array}$ & $\begin{array}{l}\text { ICV (Poland) } \\
\text { PIF (India) }\end{array}$ & $\begin{array}{l}=6.630 \\
=1.940\end{array}$ \\
\hline
\end{tabular}

выполнения процесса) значение некоторого производного показателя процессов.

На данном этапе для всех отслеживаемых производных показателей предлагается экспертным путем устанавливать шкалы их измерения, включающие целевое значение, а также допустимые границы изменения данного показателя:

- верхнюю границу и (или) нижнюю границу;

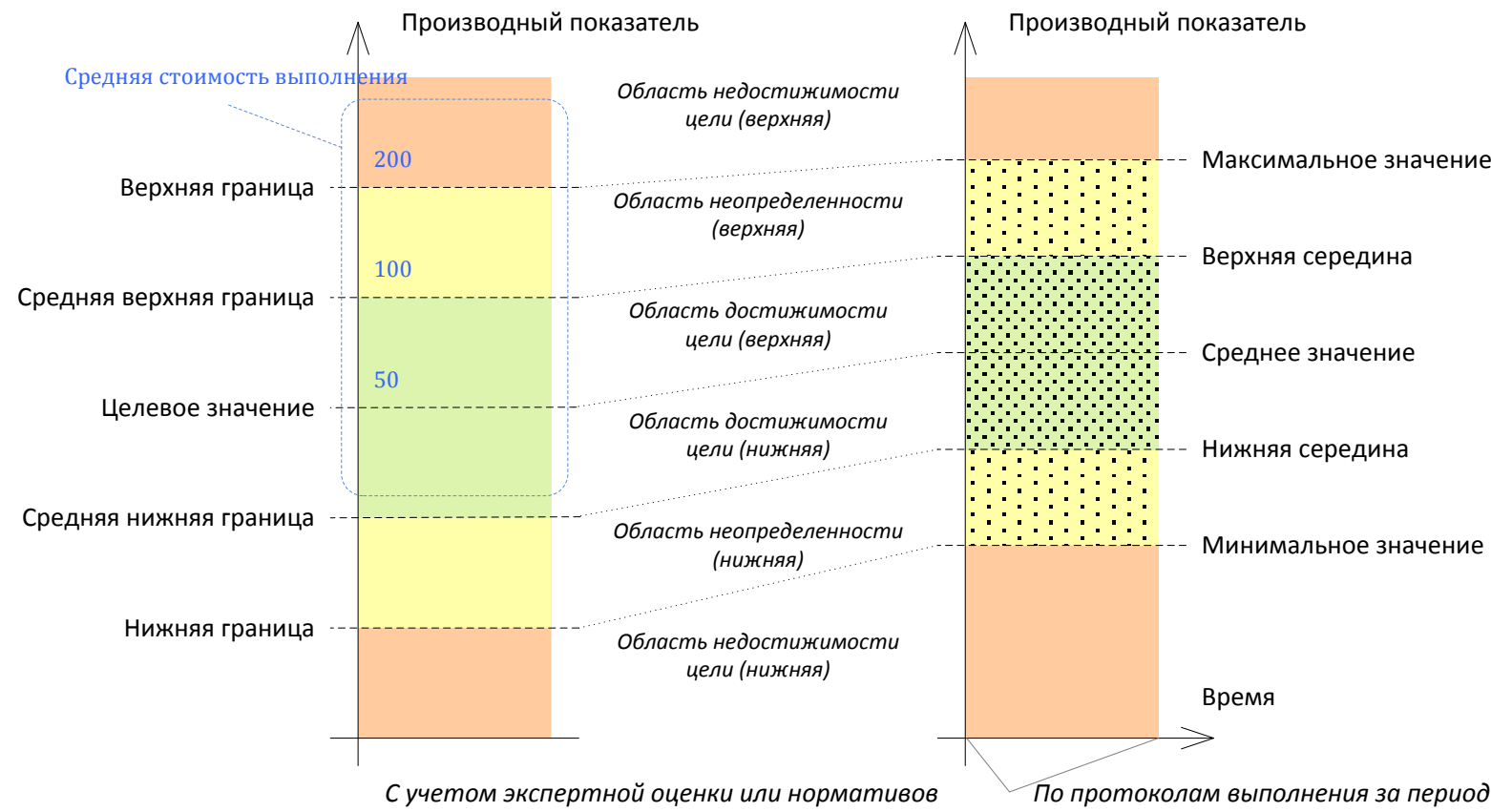

Рисунок 2 - Шкалы измерения производных показателей.

Целевое значение отражает нормативное или оптимальное значение показателя, при котором процесс выполняется в установленном режиме, т. е. без нарушения регламента. А отклонение от целевого значения в пределах средних границ отражает, выполняется ли деятельность в допустимом порядке или нет - с незначительными или редкими нарушениями регламента. В общем виде шкала измерения $l-$ го производного показателя для процесса $P_{n}$ определяется следующим образом:

$$
s c_{n, l}=\left(v_{n, l}^{\text {low }} ; v_{n, l}^{\text {mid.low }} ; v_{n, l}^{\text {mid }} ; v_{n, l}^{\text {mid.up }} ; v_{n, l}^{u p}\right)
$$

Приведем пример задания целей и шкалы для производных показателей процессов. Для процесса по приему заказа целевое значение показателей Prt $_{o R \text {,success }}$ может равняться 0.25 (работ в час) и средней стоимости приема заказа $P f v_{o R}-50$ (денежных единиц за работу), а их шкалы измерения:
- среднюю верхнюю границу и (или) среднюю нижнюю границу.

За верхней (нижней) границей образуется область недостижимости цели по данному показателю, за средней верхней (нижней) границей - неопределенности достижимости цели, а между средними границами - область ее достижимости, как показано на рисунке 2 (шкала измерения показателя слева). 


$$
\begin{gathered}
v_{n, l}^{\text {mid.up }}=v_{n, l}^{\text {mid }}+d v_{n, l}^{\text {aver }}, \\
v_{n, l}^{u p}=v_{n, l}^{\text {mid }}+d v_{n, l}^{\max } .
\end{gathered}
$$

Помимо такого ручного способа установки целей для производных показателей при их определении могут использоваться этих показателей за какой-либо успешный период работы (исходя из протоколов их выполнения в ВРМ-системе), как показано на рисунке 2 (шкала измерения показателя справа).

При этом верхней границе соответствует максимальное из всех предыдущих значений рассматриваемого показателя; нижней границе минимальное значение; целевому значению среднее значение; средней верхней границе верхняя середина; средней нижней границе нижняя середина:

$$
\begin{gathered}
v_{n, l}^{u p}=\max _{\{i\}}\left(Y_{n, l}^{i}\right), \\
v_{n, l}^{\text {low }}=\min _{\{i\}}\left(Y_{n, l}^{i}\right), \\
v_{n, l}^{\text {mid }}=\underset{\{i\}}{\operatorname{avg}\left(Y_{n, l}^{i}\right),} \\
v_{n, e}^{\text {mid.up }}=\operatorname{avg}\left(v_{n, l}^{\text {mid }} ; v_{n, l}^{\text {up }}\right), \\
v_{n, e}^{\text {mid.low }}=\operatorname{avg}\left(v_{n, l}^{\text {low }} ; v_{n, l}^{\text {mid }}\right) .
\end{gathered}
$$

В результате задания целевых значений и шкал измерения производных показателей процессов определяются возможности по достижимости целей при управлении работой.

Шаг 3. Определение контекста для управления операционными рисками на основе исходных показателей процессов

В общем случае оперативное управление в организационно-технических системах связано с установлением фактов и вычислением возможности возникновения каких-либо потерь, выражаемых в денежном, качественном или другом виде, и последующим реагированием на них. В теории управления рисками для обозначения рисков, отражающих нарушение регламента выполнения бизнес-процессов компании, источниками которого могут являться оборудование, сотрудники, внешние события нефинансовой природы, методическая ошибочность самого регламента коллективной деятельности [9], используется понятие ОР.

В [10] предложена идея реализации управления ОР за счет комплексного описания всех бизнес-процессов компании с одновременным выделением узких мест как источников риска в каждом из них, знания о которых при выполнении деятельности можно использовать для анализа ОР с целью принятия решений о регулировании бизнес-процессов или стратегии по управлению рисками. При этом сами ОР целесообразно связывать с различными событиями, возникающими при исполнении процесса (получение сигнала или сообщения, завершение, останов, ошибка и т. д.) [11, с. 102].

При этом возможность автоматизации оценки OP на основе учета таких событий обеспечивается использованием формализованных моделей деятельности, а также описания связей всех сущностей процессного управления, позволяющих учитывать расход ресурсов при исполнении этих моделей.

Еще один вариант определения рисков при управлении бизнес-процессами заключается в оценке показателей их эффективности, рассчитываемых на основании векторов входных, внутренних и выходных ресурсов, заданных с некоторой неопределенностью [12], но без учета операционной составляющей деятельности. Этот способ позволяет учитывать финансовую составляющую процессов для обоснования выбора предпочтения портфелей процессов.

Также риск может быть определен посредством рассмотрения составной рискситуации, обобщающей возможности проявления, последствия и значимость отдельных риск-событий, ведущих к его возникновению [13]. В данном методе ОР предлагается связывать с такими событиями, которые отражают нарушение «нормального» хода выполнения деятельности.

Тогда ОР, возникающий в ходе реализации коллективной деятельности, выполняемой на основе их формализованных моделей, можно определить в виде свертки возможностей возникновения отдельных негативных событий источников риска:

$$
R_{n, r}=\operatorname{operator}_{\{n, j\}}\left(r s_{n, j}\right),
$$

где operator - оператор для расчета ОР, учитывающий несколько риск-событий; rиндекс риск-ситуации; $j$ - индекс учитываемого неблагоприятного события, возникающего при выполнении $P_{n}$. Источником риска при этом может быть собственно процессное события $e$ или событие, связанное с учетом расхода ресурса $y$.

В качестве оператора для расчета ОР, проводящего свертку возможностей проявления риск-событий, предлагается применить среднее арифметическое, минимум или максимум:

$$
\text { operator }=\{a v g, \min , \max \},
$$

выбор которых зависит от характера источников OP [14], для которых при рассмотрении:

- событий, дестабилизирующих деятельность (содержащих угрозы невыполнения), используется операция $\max$, определяющая угрозу с максимальной возможностью реализации; 
- событий, снижающих результативность, но не дестабилизирующих деятельность, используется операция $\mathrm{avg}$;

- событий, имеющих негативные последствия (какой-либо ущерб для организации), не связанные с деятельностью, используется операция типа min.

В общем случае риск-событие вычисляется в виде произведения значимости последствий и «вероятности» его наступления, что можно интерпретировать как:

$$
r s_{n, j}=k_{n, j} \cdot \operatorname{Pri}_{n, j}
$$

где $k_{n, j}$ - значимость последствий события; $\operatorname{Pr} i_{n, j}$ - относительная частота наступления события (см. шаг 1) исходя из статистики выполнения $P_{n}$.

Исходя из того, что в данном выражении в количественном виде не учитывается мера достижимости цели, для определения ОР возможно использовать не статистический, а эффективный риск проявления его отдельных негативных событий, рассчитываемый неформально, через некоторую меру, когда это возможно. В качестве нее предлагается принять меру отклонения показателя, полученного для неблагоприятного события или при учете расходования ресурса относительно заданной нормы:

$$
\begin{gathered}
d y_{n, j}=\left\{\begin{array}{lr}
d y_{n, e}, & \text { для негативного события, } \\
d y_{n, y}, & \text { при учете расхода ресурса, }
\end{array}\right. \\
d y_{n, e}=\frac{Y_{n, e}}{\overline{Y_{n, e}}}, d y_{n, y}=\frac{\left|Y_{n, y}-\overline{Y_{n, y}}\right|}{\overline{Y_{n, y}}} .
\end{gathered}
$$

где $\overline{Y_{n}}$ - нормативное значение показателя $Y_{n}$.

При отсутствии значений этой нормы мера $d y_{n, e}$ рассчитывается как статистический риск (через показатель $P r i_{n, e}$ ), а мера $d y_{n, y}-$ в виде:

$$
d y_{n, y}=\frac{\left|Y_{n, y}-Y_{n}\right|}{Y_{n}}
$$

Поскольку мера отклонения показателя будет использоваться при свертке возможностей нескольких риск-событий, она должна быть нормирована. С учетом этого ограничения, рисксобытие определим как произведение коэффициента значимости его последствий и меры отклонения показателя, не превышающей $\ll 1 »$ :

$$
r s_{n, j}=\left\{\begin{array}{c}
k_{n, j} \cdot d y_{n, j}, \text { если } d y_{n, j}<1, \\
k_{n, j}, \text { если } d y_{n, j} \geq 1
\end{array}\right.
$$

Согласно этому выражению возможность проявления негативного события определяется с использованием показателей деятельности, отражающих данные не только о фактическом их наступлении, но и количественной мере их проявления относительно нормального порядка выполнения процесса.

При этом для первоочередного отслеживания самых неблагоприятных событий определяются коэффициенты значимости последствий их наступления (например, экспертным путем), иначе считается, что риск имеет значимые последствия $-k_{n, j}=1$.

C учетом этого для рассматриваемого примера опишем следующие ОР.

Риск снижения оперативности приема заказа, учитывающий отдельные «узкие места» по средним временам выполнения процессов $P_{\text {reqsend }}$ всеми сотрудниками и $P_{\text {ordHandl }}$ не основными специалистами, рассчитывается с использованием оператора свертки, снижающих результативность:

$$
\begin{gathered}
R_{\text {oR,opDec }}=\operatorname{avg}\left(r s_{r S n d} ; r s_{\text {oHnd } / \text { clrk }}\right), \\
r s_{r S n d}=k_{r S n d} \cdot \frac{\left|R s t_{r S n d}-\overline{R s t_{r S n d}}\right|}{\overline{R s t_{r S n d}}}, \\
r s_{o H n d / c l r k}=k_{o H n d / c l r k} \cdot \frac{\left|R s t_{o H n d / c l r k}-R s t_{o H n d}\right|}{R s t_{o H n d}},
\end{gathered}
$$

где $\overline{R s t}_{r S n d}$ - норма продолжительности процесса $P_{\text {reqsend }}$.

Риск несогласования заказа, учитывающий долю несогласованных заказов при выполнении $P_{\text {ordRec }}$, определяется как:

$$
R_{o R, n o t A c c}=k_{o R, n o t A c c} \cdot \frac{I_{o R, n o t A c c}}{\overline{I_{o R, n o t A c c}}},
$$

где $I_{o R, n o t A c c}^{n m}$ - норма несогласований заказа при выполнении $P_{\text {ordRec }}$.

Риск чрезмерного расходования материала определяется через отклонение от нормы:

$$
R_{\text {oHnd } / M}=k_{o H n d / M} \cdot \frac{\mid \text { Prm }_{\text {oHnd } / M}-\overline{\text { Prm }_{\text {oHnd } / M}} \mid}{\overline{P r m_{o H n d / M}}},
$$

где $\overline{P r m_{o H n d / M}}$ - норма расхода материала $M$ при выполнении $P_{\text {ordHandl }}$.

Риск отклонения предложения клиентом при выполнении P ordRec рассчитывается как статистический риск, поскольку влиять на него процессными методами невозможно:

$$
R_{o R, n o t A r r}=k_{o R, n o t A r r} \cdot \operatorname{Pr} i_{o R, n o t A r r} .
$$

Значимость рисков для примера установим через следующие коэффициенты:

$$
\begin{gathered}
k_{o H n d / M}=0.8, k_{o R, \text { not Arr }}=0.2, \\
k_{o R, n o t A c c}=0.6, k_{o H n d / \text { clerk }}=0.9 .
\end{gathered}
$$

Далее, для каждого отслеживаемого производного показателя процессов ставятся в соответствие определенные выше риски: 


$$
\begin{gathered}
A_{\text {risk }}=\left\{a_{\text {risk }}\left(Y_{n, l}\right)\right\}, \\
a_{\text {risk }}\left(Y_{n, l}\right)=\left\{Y_{n, l} \rightarrow\left\{R_{n, r}\right\}\right\} .
\end{gathered}
$$

Так, показателям производительности и средней стоимости приема заказа будут соответствовать ОР, заданные в выражениях:

$$
\begin{gathered}
A_{\text {risk }}=\left(a_{\text {risk }}\left(P r i_{o R}\right), a_{\text {risk }}\left(P f v_{o R}\right)\right), \\
a_{\text {risk }}\left(P r t_{o R, \text { success }}\right)=\left(P r i_{o R} \rightarrow\left(\begin{array}{c}
R_{\text {oR,notArr }}, \\
R_{\text {oR,notAcc }} \\
R_{\text {oR,opDec }}
\end{array}\right)\right), \\
a_{\text {risk }}\left(P f v_{o R}\right)=\left(P f v_{o R} \rightarrow\left(\begin{array}{l}
R_{\text {oHnd } / M}, \\
R_{\text {oR,opDec }}
\end{array}\right)\right) .
\end{gathered}
$$

По результатам шага 3 для производных показателей устанавливаются наименования и выражения для определения ОР на основе значений исходных показателей процессов $\mathrm{c}$ учетом характера риск-событий и норм их выполнения в случае необходимости.

Шаг 4. Задание компенсирующих воздействий и предупредительных мер для профилактики операционных рисков

На основании данных об операционных рисках для тех или иных неблагоприятных ситуаций затем задаются компенсирующие воздействия, корректирующие мероприятия и предупредительные меры для их профилактики.

Перечень этих допустимых мероприятий определяется исходя из данных о сущностях, на которые требуется воздействовать при оперативном управлении работой, - ресурсах (трудовых, материалов, оборудования) и собственно процессах $[4$, с. $357 ; 5 ; 8]$. Исходя из этого, для осуществления управления предлагаются категории мероприятий для

\begin{tabular}{|c|c|c|c|}
\hline \multicolumn{3}{|c|}{$\begin{array}{c}\text { Категория сущностей и типы } \\
\text { воздействий }\end{array}$} & $\begin{array}{c}\text { Компенсирующие воздействия и меры для профилактики } \\
\text { операционных рисков }\end{array}$ \\
\hline \multirow{6}{*}{ 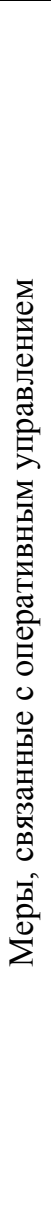 } & \multirow{3}{*}{ 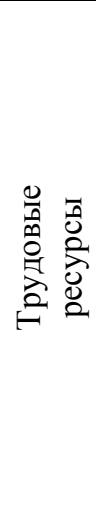 } & $\begin{array}{l}\text { Управление } \\
\text { сотрудниками }\end{array}$ & $\begin{array}{l}\text { - выбор кандидатов для выполнения задания (по их загруженности } \\
\text { (минимизация времени ожидания следующего задания), по опыту } \\
\text { выполнения, по способностям) [5]; } \\
\text { - регулирование (увеличение, уменьшение числа) участвующих в } \\
\text { работе сотрудников; } \\
\text { - замена исполнителя текущего задания или кандидатов последующих } \\
\text { для заданий (в рамках модели организации деятельности); }\end{array}$ \\
\hline & & Учет должностей & $\begin{array}{l}\text { - воздействие на выбор кандидатов по должности (по старшинству, в } \\
\text { обратном порядке) [5]; }\end{array}$ \\
\hline & & $\begin{array}{l}\text { Учет } \\
\text { организационных } \\
\text { единиц }\end{array}$ & $\begin{array}{l}\text { - воздействие на выбор кандидатов по уровню организационной } \\
\text { единицы (по иерархии, в обратном порядке); }\end{array}$ \\
\hline & \multirow{2}{*}{ 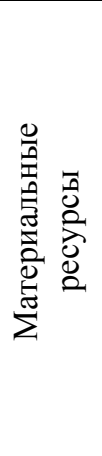 } & $\begin{array}{l}\text { Управление } \\
\text { расходованием } \\
\text { материалов }\end{array}$ & $\begin{array}{l}\text { - регулирование расходования материалов (увеличение, уменьшение) } \\
\text { по количеству или стоимости их использования для выполнения } \\
\text { процесса; } \\
\text { - замена типов используемых материалов; }\end{array}$ \\
\hline & & $\begin{array}{l}\text { Управление } \\
\text { использованием } \\
\text { оборудования }\end{array}$ & $\begin{array}{l}\text { - определение порядка использования оборудования (минимизация } \\
\text { числа переключений оборудования, минимизация простоя } \\
\text { оборудования); } \\
\text { - регулирование числа задействованного оборудования (увеличение } \\
\text { или уменьшение количества типов или конкретных единиц); } \\
\text { - замена типов или конкретных единиц задействованного } \\
\text { оборудования; }\end{array}$ \\
\hline & 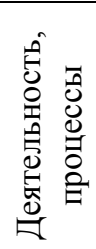 & $\begin{array}{l}\text { Управление } \\
\text { процессом }\end{array}$ & $\begin{array}{l}\text { - определение порядка выполнения заданий (понижение, повышение } \\
\text { приоритетности задания); } \\
\text { - изменение хода выполнения процесса (в рамках модели процесса); } \\
\text { - завершение работы (остановка); }\end{array}$ \\
\hline
\end{tabular}
устранения рисков и их последствий, как показано в таблице 2.

Таблица 2

Категории компенсирующих воздействий и мер для профилактики ОР. 


\begin{tabular}{|l|l|}
\hline & - подготовка новых предложений и акций для клиентов; \\
& - информирование клиента о ходе работы; \\
& - стимулирование сотрудников и др. \\
& \\
\hline
\end{tabular}

Меры, связанные с оперативным управлением работой полагают регулирование трудовых и материальных ресурсов (выбор наиболее подходящих и определение порядка их использования, изменение количества или замену ресурсов), а также воздействие на выполнение экземпляров процессов со стороны их владельцев. Для реализации этих мер могут использоваться способы выбора кандидатов для выполнения и определения порядка выполнения заданий, предложенные в [5].

Напротив этому, меры не связанные с оперативным управлением, подразумевают действия, в ходе которых не осуществляется прямое воздействие на ресурсы и другие сущности процессного управления, однако которые могут служить для профилактики ОР. На них не будем останавливаться.

В данной работе также не рассматриваются меры, не относящиеся к оперативному управлению. Эти значимые для реализации процессного управления мероприятия связаны с оптимизацией бизнес-процессов и обеспечением организации деятельности, заключаются в изменении или уточнении технологического процесса и, соответственно, модели процесса, регулировании организации деятельности (за счет переопределения функций, должностей, сотрудников, а также перенастройки связей других сущностей процессного управления, определяющих профили и роли).

На основании этих корректирующих мероприятий для всех определенных на предыдущем шаге рисков формируются соответствующие планы реагирования [13], представляющие упорядоченные в порядке значимости наборы мер с параметрами их выполнения, задаваемыми в лингвистическом виде. Например, значения параметра для мероприятия по регулированию количества задействованных единиц оборудования могут определять уменьшение или увеличение их числа:

$$
c_{\text {eqReg }}=\{\text { redNum, incNum }\},
$$

где redNum - значение, определяющее уменьшение, а incNum - увеличение числа задействованных единиц оборудования.

В общем виде планы реагирования записываются следующим образом:

$$
\begin{gathered}
A_{p r v}=\left\{a_{p r v}\left(R_{n, r}\right)\right\} \\
a_{p r v}\left(R_{n, r}\right)=\left\{R_{n, r} \rightarrow\left\{\operatorname{prv}_{\text {type }}\left(c_{\text {name }}\right)\right\}\right\} .
\end{gathered}
$$

где $p r v_{\text {type }}$ - наименование мероприятия для устранения OP; $c_{\text {name }}$ - значение параметра для этого типа мероприятия.

Для нашего примера можно предложить следующие компенсирующие воздействия:

$$
\begin{aligned}
& A_{\text {prv }}=\left(a_{\text {prv }}\left(R_{\text {oHnd } / M}\right), a_{\text {prv }}\left(R_{\text {oR,opDec }}\right), a_{\text {prv }}\left(R_{\text {oR,notAcc }}\right), a_{\text {prv }}\left(R_{o R, n o t A r r}\right)\right), \\
& a_{\text {prv }}\left(R_{\text {oHnd } / M}\right)=\left(R_{\text {oHnd } / M} \rightarrow\left(\operatorname{prv}_{\text {matSel }}(\text { byMinCons }), \operatorname{prv}_{\text {eqSwitch }}(\text { byMinCycl })\right)\right),
\end{aligned}
$$

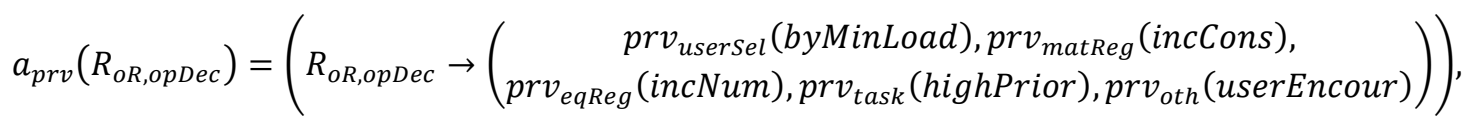

$$
\begin{aligned}
& a_{\text {prv }}\left(R_{o R, n o t A c c}\right)=\left(R_{o R, n o t A c c} \rightarrow\left(\operatorname{prv}_{\text {task }}(\text { highPrior }), \operatorname{prv}_{\text {userChang }}(\operatorname{successively)}), \operatorname{prv} v_{\text {oth }}(\operatorname{progrInf})\right)\right), \\
& a_{\text {prv }}\left(R_{\text {oR,notArr }}\right)=\left(R_{\text {oR,notArr }} \rightarrow \operatorname{prv}_{\text {oth }}(\text { newOffer })\right),
\end{aligned}
$$

где $p r v_{\text {matsel }}$ - мероприятие по замене типов материалов; prv matReg - по регулированию расходования материалов; prv $v_{\text {usersel }}$ - выбору кандидатов для выполнения заданий;

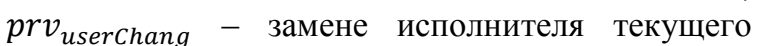
задания; prv eqReg $_{\text {R }}$ - по регулированию использования оборудования; prveqswitch управлению порядком использования оборудования; prv $v_{\text {task }}$ - определению порядка выполнения заданий; $p r v_{o t h}-$ другие меры. 
Исходя из этого, допустимые мероприятия для профилактики операционных рисков можно определить в виде множества:

$$
\operatorname{Prv}=\left(\begin{array}{c}
p r v_{\text {userReg }}, p r v_{\text {userSel }}, p r v_{\text {userChang }}, \\
p r v_{\text {matReg }}, p r v_{\text {matSel }}, p r v_{\text {matChang }} \\
p r v_{\text {eqReg }}, p r v_{\text {eqSel }}, p r v_{\text {eqSwitch }} \\
p r v_{\text {task }}, p r v_{\text {oth }}
\end{array}\right) .
$$

где $p r v_{\text {userReg }}$ - мероприятие по регулированию числа сотрудников; prv matchang - замене материалов; prveqsel - регулированию числа задействованного оборудования.

Таким образом, на данном шаге осуществляется задание наборов упорядоченных допустимых мероприятий для конкретных ОР, в том числе - установка необходимых или введение новых значений параметров этих мероприятий, а в результате выполнения этапа 1 - всех параметров для оперативного управления работой.

\section{Этап 2. Распределение ресурсов для} выполнения коллективной деятельности

На этапе 2 проводится распределение ресурсов, требуемых для выполнения коллективной деятельности. Причем выделение материалов, оборудования, трудовых ресурсов при этом осуществляется владельцем процесса для каждого типа или экземпляра процесса, как описано далее (шаг 5).

Шаг 5. Выделение необходимых ресурсов для выполнения процессов

Выделение необходимых ресурсов заключается в установлении наименований и количества единиц конкретного вида ресурса, который задействуется, используется или расходуется при выполнении рассматриваемого на данном шаге процесса.

Определение наименований ресурсов проводится согласно связям сущностей процессного управления, заданным при организации коллективной деятельности [5] для всей системы (организации):

$$
A=\left(\begin{array}{c}
A_{\text {sub }}, A_{\text {pos }}, A_{\text {cand }}, \\
A_{\text {task }}, A_{\text {func }}, A_{\text {abil }}, \\
A_{\text {set }}, A_{\text {mat }}, A_{\text {equip }}
\end{array}\right)
$$

из которых здесь используются множества связей $A_{\text {cand }}$ - замещения должностей; $A_{\text {func - }}$ реализации функций; $A_{\text {task }}$ - связей заданий и должностей; $A_{\text {abil }}$ - способностей сотрудников; $A_{\text {equip }}$ - заданий и задействованного при этом оборудования; $A_{\text {mat }}$ - заданий и используемых при этом материалов.

Эти множества, на примере $A_{\text {mat }}$, описываются в виде набора связей заданий и материалов некоторых типов, используемых при выполнении процесса:

$$
A_{\text {mat }}=\left\{a_{\text {mat }, n}\right\}, a_{\text {mat }, n}=\left(P_{n} \rightarrow\{M\}\right) .
$$

Задание параметров выделения ресурсов, т. е. количества единиц ресурса - материалов, оборудования и сотрудников, замещающих должности, выполняется для каждого процесса следующим образом:

$$
\begin{gathered}
a l_{n}=\left(a l_{n / M} ; a l_{n / H} ; a l_{n / E}\right), a l_{n / M}=\left\{a l v_{n / M}\right\}, \\
a l_{n / H}=\left\{a l v_{n / H}\right\}, a l_{n / E}=\left\{a l v_{n / E}\right\},
\end{gathered}
$$

где по каждому типу ресурса указывается числовое значение выделяемых для выполнения $P_{n}$ материалов некоторого типа $-v_{n / m}$; единиц оборудования $-v_{n / H}$; сотрудников замещающих должность $-v_{n / E}$.

Задание расхода ресурсов для экземпляра процесса в случае необходимости задается аналогично:

$$
\begin{gathered}
a l_{n}^{i}=\left(a l_{n / M}^{i} ; a l_{n / H}^{i} ; a l_{n / E}^{i}\right), a l_{n / M}^{i}=\left\{a l v_{n / M}^{i}\right\}, \\
a l_{n / H}^{i}=\left\{a l v_{n / H}^{i}\right\}, a l_{n / E}^{i}=\left\{a l v_{n / E}^{i}\right\} .
\end{gathered}
$$

При возврате к этапу 2 (после выполнения этапа 4) проводится корректировка значений этих параметров выделения ресурсов.

\section{Этап 3. Мониторинг хода выполнения и} контроль операционных рисков процессов

На этом этапе проводится мониторинг хода выполнения процессов и контроль ОР с целью принятия решений по управлению работой выполнению тех или иных корректирующих мероприятий.

Мониторинг хода выполнения заключается в анализе изменения текущих значений производных показателей процессов, которые при реализации ВРМ-подхода измеряются и фиксируются на каждой итерации.

Далее, на основании анализа отклонения значений этих показателей за установленные границы осуществляется оценка возможности проявления выявленных рисков, и затем их обработка, обеспечивающая выработку (выбор наименований и параметров) корректирующих мероприятий по распределению ресурсов.

\section{Шаг 6. Анализ изменения производных} показателей процессов

На данном шаге для текущей итерации функционирования системы для владельцев текущих процессов проводятся автоматический сбор и расчет производных показателей, а затем на основании этого - анализ их изменения.

При автоматизированном анализе также осуществляется непрерывное отображение каждого показателя $Y_{n, l}$ в графическом виде (на 


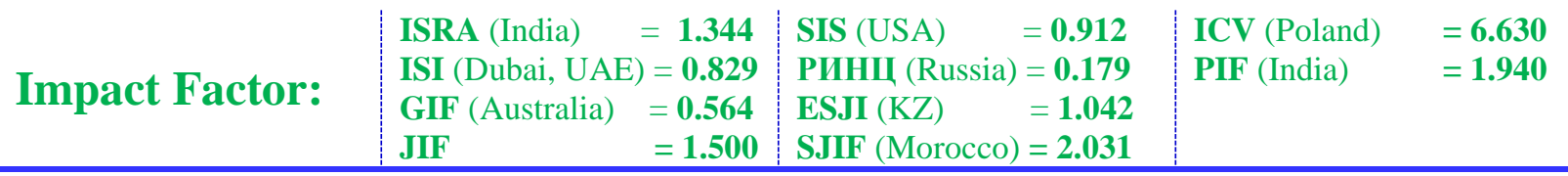

фоне соответствующей шкалы $s c_{n, l}-$ см. шаг 2): значений в зависимости от суммарного времени выполнения всех экземпляров процесса $P_{n}$.

При анализе производных показателей процессов для каждого из них проводится оценка отклонения их текущего значения от целевого значения по следующему правилу:

$$
\text { - если } v_{n, l}^{\prime}<v_{n, l}^{\text {low }} \text { или } v_{n, l}^{\prime}>v_{n, l}^{\text {up }} \text {, то }
$$

выполняется автоматическое или ручное реагирование на отклонение показателя от верхней (нижней) границы шкалы измерения вместе с информированием (переход к управлению ОР на шаге 7), иначе

$$
\text { - если } v_{n, l}^{\prime}<v_{n, l}^{\text {mid.low }} \text { или } v_{n, l}^{\prime}>v_{n, l}^{\text {mid.up }} \text {, то }
$$
осуществляется информирование владельца процесса и, опционально, выполняется ручное реагирование на отклонение показателя от средней верхней (нижней) границы шкалы измерения (возврат к шагу 6 или по усмотрению владельца процесса - переход к управлению ОР на шаге 7), иначе

$$
\text { - (если } \left.v_{n, l}^{\prime} \geq v_{n, l}^{\text {mid.low }} \text { и } v_{n, l}^{\prime} \leq v_{n, l}^{\text {mid.up }} \text {, то }\right)
$$
продолжается наблюдение за изменением данного показателя на следующей итерации функционирования системы (возврат к шагу 6).

Визуально анализ значения производного показателя процессов можно представить, как показано на рисунке 3 .

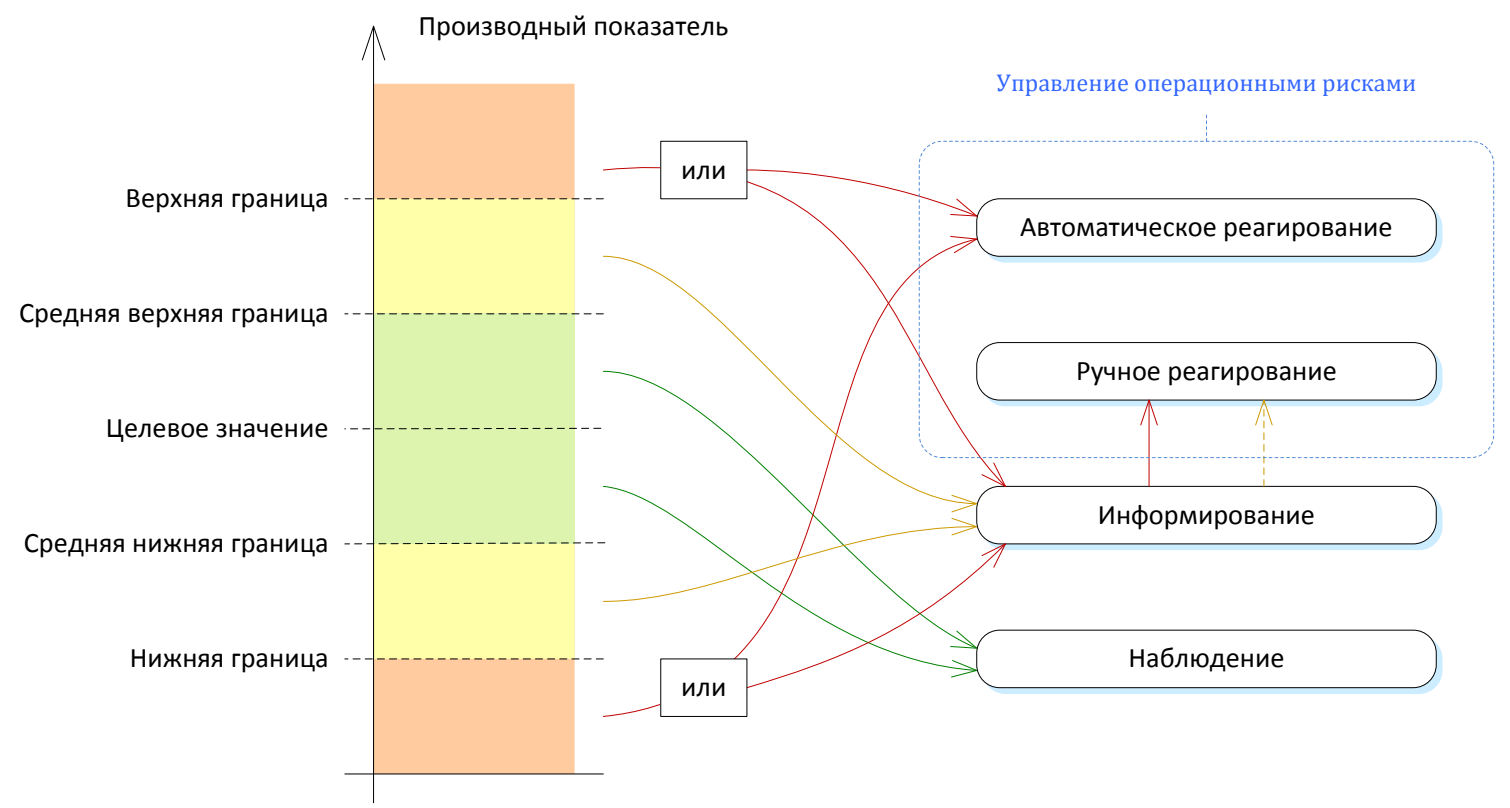

Рисунок 3 - Анализ значения производного показателя процессов.

В итоге, по результатам анализа отклонений отслеживаемых показателей от их целевых значений принимается решение о необходимости контроля операционных рисков для рассматриваемых процессов. В случае положительного решения осуществляется переход на шаг 7.

\section{Шаг 7. Оценка проявления операционных} рисков процессов

Оценка проявления операционных рисков проводится для процессов, для которых на шаге 6 выявлены существенные отклонения значений каких-либо производных показателей за средние границы (верхнюю или нижнюю границу) шкалы измерения.

Наименования оцениваемых для каждого процесса рисков устанавливаются исходя из связей типа $a_{\text {risk }}\left(Y_{n}\right)$, определяющих все риски для отслеживаемых при выполнении процесса $P_{n}$ производных показателей $Y_{n}$.

Вычисление возможности наступления ОР проводится посредством вычисления величин составляющих его риск-событий согласно выражениям, заданным для них на шаге 3 , как продемонстрировано на рисунке 4 . 


\begin{tabular}{l|lrl|l|ll} 
& ISRA (India) & $=\mathbf{1 . 3 4 4}$ & SIS (USA) & $=\mathbf{0 . 9 1 2}$ & ICV (Poland) & $=\mathbf{6 . 6 3 0}$ \\
Impact Factor: & ISI (Dubai, UAE) $=\mathbf{0 . 8 2 9}$ & PUHU (Russia) $=\mathbf{0 . 1 7 9}$ & PIF (India) & $=\mathbf{1 . 9 4 0}$ \\
& GIF (Australia) & $\mathbf{0 . 5 6 4}$ & ESJI (KZ) & $=\mathbf{1 . 0 4 2}$ & & \\
& JIF & $=\mathbf{1 . 5 0 0}$ & SJIF (Morocco) $=\mathbf{2 . 0 3 1}$ & &
\end{tabular}

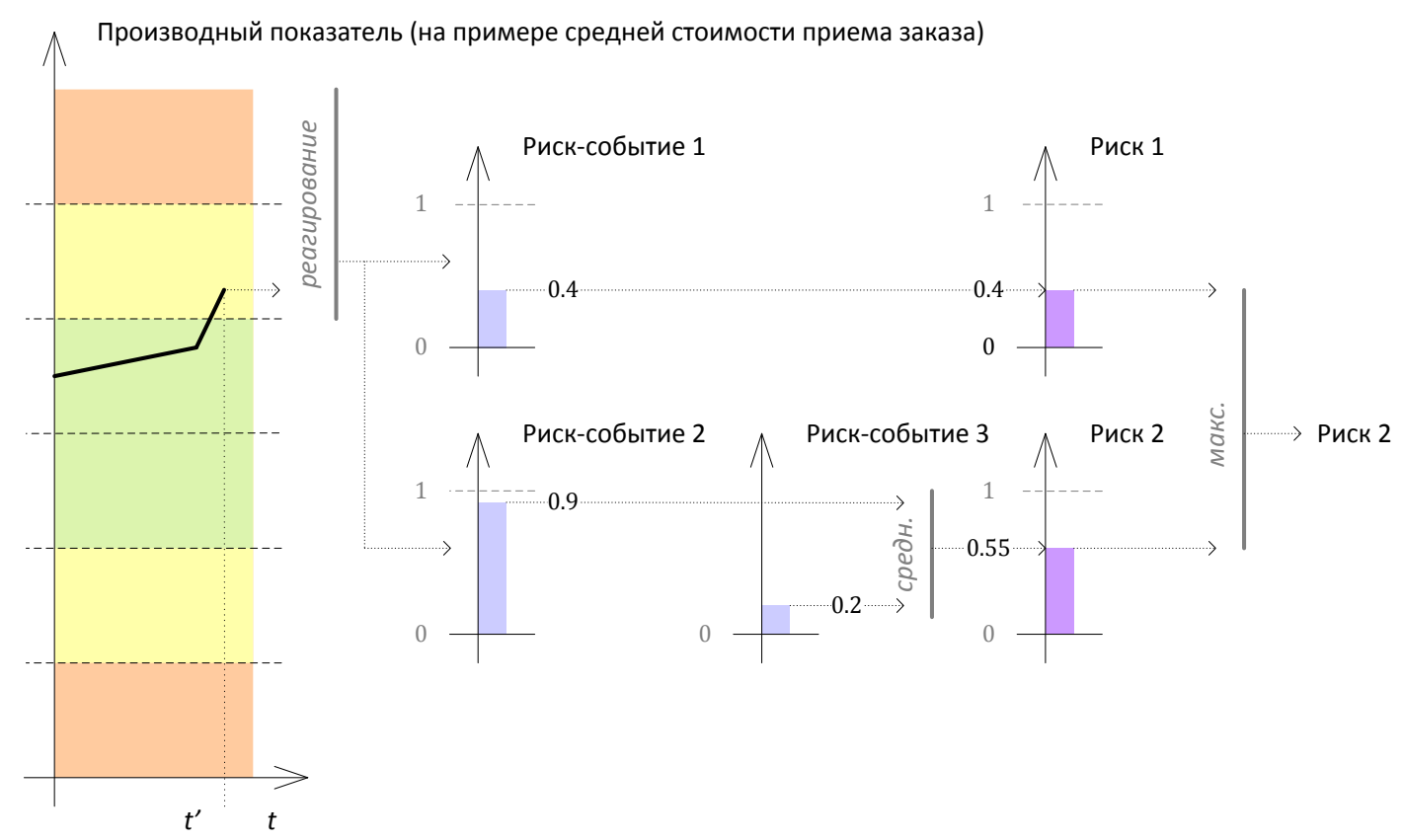

Рисунок 4 - Оценка операционных рисков в ходе реализации деятельности.

Например, при отклонении показателя средней стоимости приема заказа $P f v_{o R}$ выполняется оценка риска чрезмерного расходования материалов и риска снижения оперативности приема заказа (на рисунке 4 риск 1 и риск 2 соответственно):

$$
\begin{gathered}
r s_{o H n d / M}^{\prime}=0.8 \cdot \frac{60-40}{40}=0.4, \\
R_{o H n d / M}^{\prime}=r s_{o H n d / M}^{\prime} ; \\
r s_{r S n d}^{\prime}=1 \cdot \frac{0.6-0.5}{0.5}=0.2 ; \\
r s_{o H n d / c l r k}^{\prime}=0.9,
\end{gathered}
$$

поскольку

$$
\frac{8.5-4}{4}>1
$$

в итоге

$$
R_{o R, o p D e c}^{\prime}=\frac{r s_{r S n d}^{\prime}+r s_{o H n d / c l r k}^{\prime}}{2}=0.55 \text {. }
$$

На основании сравнения полученных значений $R_{n, r}^{\prime}$ устанавливается упорядоченный список $\left\{R_{n, r}\right\}^{\prime}$, по которому будет проводиться обработка риска:

- с максимальным значением возможности проявления;

- со значением, следующим в порядке убывания возможности проявления.
При рассмотрении этого списка на шаге 8 владельцем процесса принимается решение об обработке конкретного риска.

Приведенный подход оценки ОР «снизу вверх», адаптированный под рассматриваемую задачу, ориентирован на применение непосредственными участниками в ходе выполнения деятельности. Он, в отличие от подхода «сверху вниз», при котором ОР контролируются постфактум, например, в специализированных департаментах или группах, занимающихся классификацией, идентификацией и контролем проявления рисков в бизнеспроцессах, позволяет «управлять операционными рисками внутри функциональных линий» [10] (в процессе работы системы) на основании данных о ходе выполнении всех экземпляров рассматриваемых процессов.

Шаг 8. Обработка операционных рисков и принятие решения по регулированию ресурсов

Для рассматриваемых процессов, для которых установлен факт отклонения значения хотя бы одного из производных показателей, далее выполняется обработка всех ОР, в результате чего принимаются или не принимаются решения о необходимости регулирования ресурсов.

Процесс принятия решений по выбору корректирующих мероприятий по регулированию ресурсов на основе данных о списке $\left\{R_{n, r}\right\}^{\prime}$ включает следующие действия. Для главного 


\begin{tabular}{|c|c|c|c|c|c|c|}
\hline Impact Factor: & $\begin{array}{l}\text { ISRA (India) } \\
\text { ISI (Dubai, UAB } \\
\text { GIF (Australia) } \\
\text { JIF }\end{array}$ & $\begin{array}{r}=1.344 \\
=0.829 \\
=0.564 \\
=1.500\end{array}$ & $\begin{array}{l}\text { SIS (USA) } \\
\text { PИHЦ (Russia) } \\
\text { ESJI (KZ) } \\
\text { SJIF (Morocco) }\end{array}$ & $\begin{array}{l}=0.912 \\
=0.179 \\
=1.042 \\
=2.031\end{array}$ & $\begin{array}{l}\text { ICV (Poland) } \\
\text { PIF (India) }\end{array}$ & $\begin{array}{l}=6.630 \\
=1.940\end{array}$ \\
\hline
\end{tabular}

риска, имеющего максимальную возможность проявления, все заданные для него корректирующие мероприятия рассматриваются в порядке, указанном экспертом в соответствующем плане реагирования $a_{p r v}\left(R_{n, r}\right)$ на данный риск, и группируются по категориям ресурсов.

При этом для других рисков, с меньшей возможностью проявления, корректирующие мероприятия рассматриваются в порядке, согласованном по категориям ресурсов с порядком мероприятий главного риска, как показано на рисунке 5.
Так, рассмотрение мероприятий $\operatorname{pr} v_{\text {usersel }}$, $p r v_{\text {matReg }}, p r v_{\text {eqReg }}, p r v_{\text {task }}, p r v_{\text {oth }}$, заданных в плане $a_{p r v}\left(R_{o R, o p D e c}\right)$ для риска снижения оперативности приема заказа, если он является главным, выполняется в следующем порядке для категорий мероприятий:

- управление трудовыми ресурсами;

- управление материалами;

- управление оборудованием;

- управление процессами;

- другие мероприятия.

Риски в порядке убывания возможности их возникновения в процессе $n$

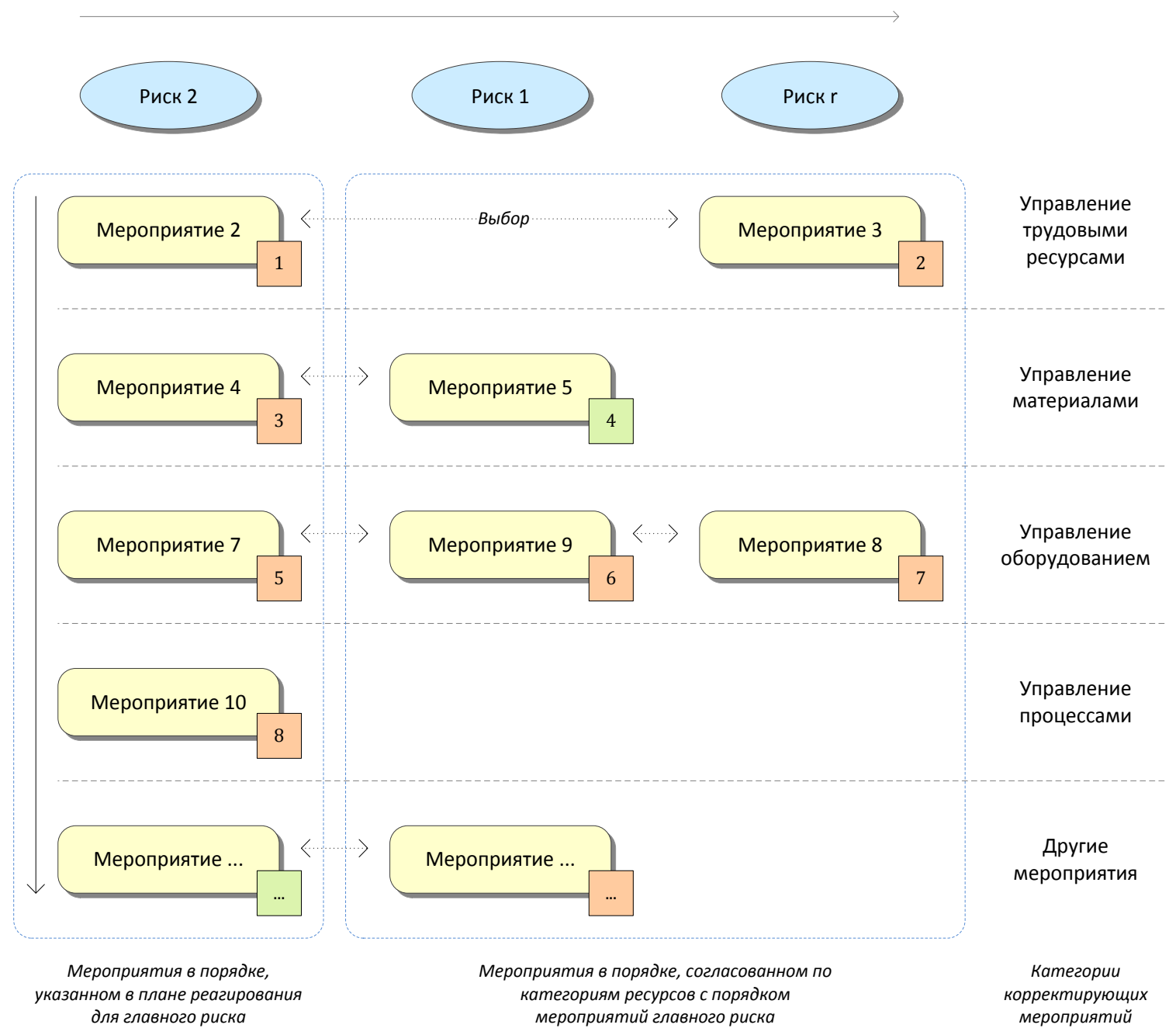

Рисунок 5 - Выбор корректирующих мероприятий по регулированию ресурсов.

Далее для каждой категории проводится сравнение и выбор не более одного мероприятия из тех, что направленны на управление конкретным видом ресурса для всех существующих рисков для данного процесса, поскольку выполнение над одним ресурсом различных по характеру корректирующих мероприятий не обеспечит предсказуемость результатов обработки всех рисков. Критерием выбора мероприятия для каждой категории 
может служить минимизация стоимости расхода pecypca, который предполагается выделить (согласно данным $a l_{n}$ ) в результате выполнения сравниваемых мероприятий.

В отдельных случаях, когда возможно появление трудностей, не позволяющих применять мероприятие, предлагаемое согласно критерию минимизации стоимости расхода peсурса, выбор может осуществляться по усмотрению владельца процесса (ручным способом).

Например, если при реагировании на повышение средней стоимости приема заказа не одно из мероприятий по управлению трудовыми ресурсами невозможно выполнить (prv $v_{\text {usersel }}$,

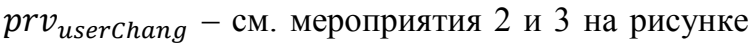
6), то осуществляется переход к рассмотрению мероприятий по управлению материалами $\left(p r v_{\text {matReg }}\right.$, prv $v_{\text {matsel }}$ - см. мероприятия 4 и 5 на рисунке 6). При этом может случиться, что в конкретном случае владельцу процесса целесообразно принять мероприятие 5 (по замене используемого для данного задания типа материала), а не мероприятие 4 (по увеличению расходования материалов текущего типа), заданное в плане реагирования для данного типа риска.

Также данный пример демонстрирует невозможность применения различных по характеру мероприятий для регулирования одного и того же ресурса.

В результате рассмотрения всех категорий по порядку осуществляется:

- выбор корректирующих мероприятий по регулированию ресурсов на основании данных о возможных ОР и составленных заранее планах реагирования процесса;

- затем, при необходимости, выполнение этих мероприятий (переход к этапу 2).

Очевидно, что планы реагирования при этом должны быть составлены экспертом таким образом, чтобы в большинстве случаев при отклонении значений производных показателей при обработке наиболее возможных рисков выполнялись те мероприятия, что указаны раньше. Это позволит проводить обработку ОР владельцам процессов с большей степенью автоматизации.

Если после обработки ОР корректирующие мероприятия не выбраны или, по мнению владельца процесса, не требуют их выполнения, то проводится проверка завершения работы. Если исполнение коллективной деятельности завершено (требуется прекратить оперативное управление работой), то метод завершается, иначе выполняется переход к этапу 4.

Таким образом, на этапе 3 осуществляется принятие решений по оперативному управлению работой на основе контроля ОР, обеспечивающих выработку корректирующих мероприятий по распределению ресурсов для наиболее критических участков прохождения процесса на основе анализа отклонений от целевых значений тех показателей, которые фиксируются непосредственно при работе системы.

Этап 4. Анализ результатов оперативного управления работой

Этот этап выполняется при необходимости анализа результатов и корректировки параметров оперативного управления работой.

Шаг 9. Ознакомление с результатами коллективной деятельности и оперативного управления работой

На данном шаге владельцами процессов проводится ознакомление с результатами:

- коллективной деятельности в части динамики изменения, в том числе с использованием графиков и диаграмм для визуального анализа:

- отслеживаемых при контроле ОР производных показателей процессов;

- других исходных и производных показателей процессов и ресурсов (например, количества используемых единиц оборудования, среднего времени выполнения процесса всеми либо конкретным сотрудником (должностью), расхода материалов, средней стоимости работы сотрудников, средней стоимости расходования материалов при выполнении работы и т. д.);

- оперативного управления работой в части последних корректирующих мероприятий для распределения ресурсов.

После этого, в случае необходимости уточнения параметров оперативного управления работой, осуществляется переход шаг 10, иначе этап 4 завершается и выполняется возврат к этапу 3.

Шаг 10. Принятие решения об уточнении параметров для оперативного управления работой

На данном шаге, на основании анализа результатов коллективной деятельности и оперативного управления работой владельцами процессов осуществляется принятие решения об уточнении параметров для оперативного управления работой.

После этого, исходя из типа требующих уточнения параметров (показатели, цели и шкалы измерений, риски, корректирующие мероприятия), выполняется переход к соответствующему шагу (1-4) на этапе 1. 


\begin{tabular}{|c|c|c|c|c|c|c|}
\hline Impact Factor: & $\begin{array}{l}\text { ISRA (India) } \\
\text { ISI (Dubai, UAF } \\
\text { GIF (Australia) } \\
\text { JIF }\end{array}$ & $\begin{array}{l}=1.344 \\
=0.829 \\
=0.564 \\
=1.500\end{array}$ & $\begin{array}{l}\text { SIS (USA) } \\
\text { PИНЦ (Russia } \\
\text { ESJI (KZ) } \\
\text { SJIF (Morocco }\end{array}$ & $\begin{aligned}= & 0.912 \\
= & 0.179 \\
= & \mathbf{1 . 0 4 2} \\
= & \mathbf{2 . 0 3 1}\end{aligned}$ & $\begin{array}{l}\text { ICV (Poland) } \\
\text { PIF (India) }\end{array}$ & $\begin{array}{l}=6.630 \\
=1.940\end{array}$ \\
\hline
\end{tabular}

\section{Заключение}

Данный метод оперативного управления работой включает систематизированный порядок выполнения действий, разбитый на этапы настройки параметров, распределения ресурсов, мониторинга выполнения и контроля операционных рисков, а также анализа результатов оперативного управления работой, и использования способов описания формализованных моделей деятельности и оценки рисков «снизу вверх».

Особенностью метода является то, что в нем предлагается:

- определять с использованием формализованного описания деятельности и исходных показателей процессов и затрат ресурсов производные показатели (производительности и эффективности);

- рассчитывать возможность возникновения операционных рисков в виде свертки возможностей возникновения риск-событий, зависящей от их характера; $\begin{array}{ccc}\text { - учитывать } & \text { при } & \text { определении } \\ \text { неблагоприятных } & \text { событий, } & \text { отражающих }\end{array}$ нарушение хода выполнения процесса и расхода pecypca, частоту наступления этих событий или меру отклонения некоторого показателя процесса относительно заданной нормы или среднего статистического значения;

- задавать наборы упорядоченных компенсирующих мероприятий для реагирования на соответствующие операционные риски по категориям ресурсов;

- анализировать достижение целей для отслеживаемых производных показателей и контролировать соответствующие им операционные риски на уровне отдельных владельцев процессов;

- упорядочивать операционные риски по возможности их возникновения и обрабатывать согласно принадлежности предполагаемого для их устранения корректирующего мероприятия к определенной категории ресурсов;

- настраивать параметры оперативного управления работой с целью определения особых условий распределения ресурсов для выполнения коллективной деятельности на наиболее трудных участках процесса, где существуют операционные риски.

Таким образом, предложенный метод оперативного управления работой в организационно-технических системах позволяет осуществлять интеллектуальное регулирование ресурсов на уровне отдельных владельцев процессов за счет анализа изменения производных показателей, определяемых по факту исполнения формализованных моделей деятельности, а также контроля операционных рисков, учитывающих негативные события, возникающие при реализации коллективной деятельности.

Исследование выполнено при финансовой поддержке РФФИ в рамках научного проекта № 14-0700338_a, а также в рамках базовой части государственного задания Минобрнауки России № 2014/123 на выполнение государственных работ в сфере научной деятельности, проект № 2493.

\section{References:}

1. (2014) Bizagi BPM Suite - Functional Description, Bizagi Ltd., 34 p.

2. Muehlen MZ, Ting-Yi Ho D (2006) Risk Management in the BPM Lifecycle. Business Process Management Workshops. Berlin: Springer Heidelberg, vol. 3812, pp. 454-466.

3. Kuznecova ES (2010) Upravlenie operacionnymi riskami na osnove processnogo podhoda. Vestnik murmanskogo gosudarstvennogo tehnicheskogo universiteta, vol. 13 , no. 1 , pp. 63-67.

4. Repin VV (2013) Biznes-processy. Modelirovanie, vnedrenie, upravlenie. Mann, Ivanov i Ferber, 512 p.
5. Syskov VV, Borisov VV (2015) The Method of organization of collective activity in complex organizational and technical systems. Sovremennye nauchnye issledovanija i innovacii, no. 11, Available: http://web.snauka.ru/issues/2015/11/60038 (Accessed: 30.11.2015).

6. Syskov VV, Borisov VV (2015) Method of consistent description of collective activity in complex organizational and technical systems. Theoretical \& Applied Science, no. 11 (31), DOI:

http://dx.doi.org/10.15863/TAS.2015.11.31.8 


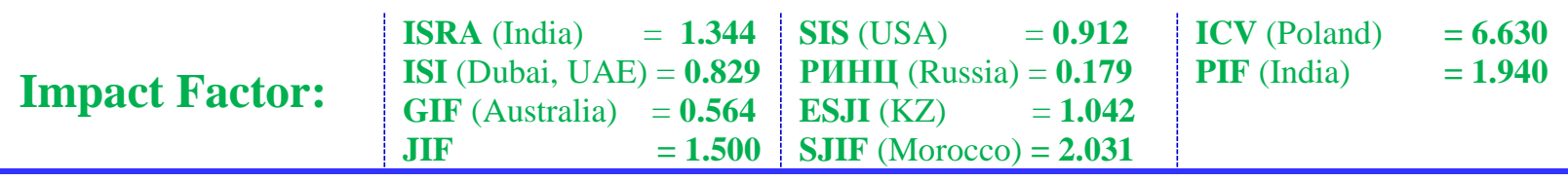

7. Kuznecov AN, Novruzlu JeA, Starostenko SV (2014) KPI-analiz kak metod antikrizisnogo upravlenija predprijatiem. Strategii biznesa, no. 2, pp. 39-42.

8. Stojanova OV, Dli MI (2015) Informacionnoanaliticheskaja sistema upravlenija proizvodstvennymi proektami mashinostroenija v uslovijah neopredelennosti. Programmnye produkty i sistemy, no. 3, pp. 49-56.

9. Shevcova JV (2010) Ponjatie operacionnogo riska predprijatija: processnyj podhod. Rossijskoe predprinimatel'stvo, no. 9, iss. 2 (167), pp. 50-55.

10. Stavenko JA, Gromov AI, Kazancev NS (2012) Podhod k upravleniju operacionnymi riskami biznes-processov organizacii. Informacionnye tehnologii $\mathrm{v}$ proektirovanii i proizvodstve, no. 3, pp. 3-10.
11. Hitpas B (2014) Business Process Management (BPM): Concepts, and How to Apply and Integrate with IT. Santiago: BPM Center, 306 p.

12. Naumov AA, Shubin DA (2008) K ocenivaniju riskov biznes-processov. Vestnik Novosibirskogo gosudarstvennogo universiteta. Serija: Social'no-jekonomicheskie nauki, vol. 8, no. 4, pp. 56-67.

13. Borisov VV, Senkov AV (2014) Models of decision-making support on the basis of riskssituations. Nechetkie sistemy i mjagkie vychislenija, vol. 9, no. 1, pp. 19-38.

14. Goncharov MM (2012) A model and a method of information security risks analysis for computer systems based on hybrid fuzzy models. Neurocomputers: Design \& Application, no. 5, pp. 9-15. 